\title{
Ultrananocrystalline diamond film as an optimal cell interface for biomedical applications
}

\author{
Piyush Bajaj • Demir Akin • Amit Gupta • \\ Debby Sherman • Bing Shi • Orlando Auciello • \\ Rashid Bashir
}

Published online: 25 May 2007

(C) Springer Science + Business Media, LLC 2007

\begin{abstract}
Surfaces of materials that promote cell adhesion, proliferation, and growth are critical for new generation of implantable biomedical devices. These films should be able to coat complex geometrical shapes very conformally, with smooth surfaces to produce hermetic bioinert protective coatings, or to provide surfaces for cell grafting through appropriate functionalization. Upon performing a survey of desirable properties such as chemical inertness, low friction coefficient, high wear resistance, and a high Young's
\end{abstract}

P. Bajaj · D. Akin · R. Bashir $(\bowtie)$

Birck Nanotechnology Center, Bindley Biosciences Center,

Purdue University, West Lafayette, IN, USA

e-mail: bashir@purdue.edu

P. Bajaj · D. Akin $\cdot$ R. Bashir

Weldon School of Biomedical Engineering, Purdue University,

West Lafayette, IN, USA

R. Bashir

School of Electrical and Computer Engineering,

Purdue University, West Lafayette, IN, USA

\section{Sherman}

Electron Microscopy Facility, Department of Biology,

Purdue University, West Lafayette, IN, USA

B. Shi $\cdot$ O. Auciello

Materials Science Division, Argonne National Laboratory,

Argonne, IL, USA

O. Auciello

e-mail: auciello@anl.gov

O. Auciello

Center for Nanoscale Materials, Argonne National Laboratory, Argonne, IL, USA

\section{A. Gupta}

BioMEMS Resource Center, Massachusetts General Hospital,

Cambridge, MA, USA modulus, diamond films emerge as very attractive candidates for coatings for biomedical devices. A promising novel material is ultrananocrystalline diamond $\left(\mathrm{UNCD}^{\circledR}\right)$ in thin film form, since UNCD possesses the desirable properties of diamond and can be deposited as a very smooth, conformal coating using chemical vapor deposition. In this paper, we compared cell adhesion, proliferation, and growth on UNCD films, silicon, and platinum films substrates using different cell lines. Our results showed that UNCD films exhibited superior characteristics including cell number, total cell area, and cell spreading. The results could be attributed to the nanostructured nature or a combination of nanostructure/surface chemistry of UNCD, which provides a high surface energy, hence promoting adhesion between the receptors on the cell surface and the UNCD films.

Keywords Ultrananocrystalline diamond · MEMS . BioMEMS · Biocompatibility · Nanomaterial $\cdot$ UNCD

\section{Introduction}

Thin film materials being investigated as coatings for integration into biosensors or implantable biomedical devices should ideally exhibit a combination of more than one or several outstanding mechanical, tribological (i.e. surface force of adhesion and/or friction), high or low electrical conductivity, and surface chemical functionalization for biopassivation or activation properties, compatible with the particular application. The purpose of this work was to investigate the degree of biocompatibility of ultrananocrystalline diamond (UNCD) films and to confirm whether UNCD may be used for biomedical applications such as implants, where cell attachment to the surface of the UNCD coatings 
needs to be promoted or for the manufacturing of biosensors were biomolecule attachment is critical for sensing.

Prior work demonstrated the application of UNCD to biosensors, via functionalization of the UNCD surface to attach DNA to it (Yang et al. 2002). For the studies discussed here, the effect of the native UNCD surface of films on the growth of different culture cell lines was studied in order to investigate the biocompatibility of UNCD at the cellular/inorganic surface interface. In addition, the performance of the UNCD surface biocompatibility was compared to two commonly used materials, namely, silicon and platinum. The main reason for investigating silicon in this comparative study was because of its dominant presence in the microelectromechanical system (MEMS) market and attempts at extension to application to bio-medical devices and bio-MEMS. In this respect, the studies discussed here were aimed at demonstrating that UNCD is a much more suitable material than silicon for fabrication of bioMEMS, since silicon cannot survive long term implantation into several organs of the human body, such as in the case of a $\mathrm{Si}$ microchip implantable in the eye, as the main component of an artificial retina to restore sight to people blinded by retina degeneration, where it is being demonstrated that UNCD bioinert encapsulation coating will be the enabler of the $\mathrm{Si}$ microchip implantation in the eye (Xiao et al. 2006) or as in the case of prosthesis such as artificial joints, where a high wear resistant coating like UNCD should provide also good cell adhesion to enhance fixation of the prosthesis in the bone. On the other hand, the comparison of UNCD with Platinum (Pt), as a biomaterial, was also targeted because $\mathrm{Pt}$ is a noble metal that has been extensively used for applications in microelectrodes for monitoring neural activity and for immunosensors for the brain (de Haro et al. 2002). For all the bio related applications discussed above, it is essential that cells have an affinity for the surface of the implanted device. The cultured cell lines used were chosen so as to investigate a range of different cells, including a cancer cell line (HeLa cells), rat pheochromocytoma cells (PC12 cell line), and osteoblasts (MC3T3 cell line).

The challenge for the development of implantable biomedical devices or biosensors based on MEMS is to identify materials with exceptional mechanical, thermal, chemical and tribological properties. Most important for its use in bioMEMS is the inertness of diamond which ensures that there will be no inflammatory response, favorable blood compatibility, and lack of cytotoxicity (Gardos 1999; Steffen et al. 2000). However, since diamond is the hardest know material available, and normally is available in expensive gem form, it is very difficult and cost inefficient to fabricate devices out of diamond. The alternative pathway demonstrated by our group is to synthesize diamond films over appropriate substrates and use micro- fabrication techniques to produce structures suitable for fabrication of MEMS/NEMS (Auciello et al. 2000).

Researchers at Argonne National Laboratory (ANL) have developed a unique process to produce phase-pure UNCD with $2-5 \mathrm{~nm}$ grains and smooth surface $(7-10 \mathrm{~nm} \mathrm{rms})$ (Gruen et al. 1994a,b, 1995; Jiao et al. 2001). The hardness, Young's modulus, refractive index, and friction properties of UNCD are approximately the same as that of natural diamond, while some of the material properties such as fracture strength for UNCD (4-5 GPa) exceed that of natural diamond (1-2 GPa) (Carlisle and Auciello 2003). As UNCD properties are essentially those of single crystal diamond, it is expected that UNCD would be biocompatible as well, as diamond biocompatibility was previously demonstrated (Freitas 2003).

\section{Materials and methods}

\subsection{Substrate preparation}

Three different substrates were used in the study: (100) p-type silicon wafer, platinum/titanium, and UNCD. The platinum/titanium substrate was obtained by sputtering $20 \mathrm{~nm}$ thick titanium as an adhesion layer on a silicon wafer and $100 \mathrm{~nm}$ thick platinum layer on top of the titanium using a Perkin Elmer 2400 Sputter System. The UNCD substrate was obtained as follows: $3-5 \mathrm{~nm}$ thick layer of UNCD was deposited on silicon wafers by microwave plasma chemical vapor deposition (MPCVD) using $\mathrm{Ar}(99 \%) / \mathrm{CH} 4$ (1\%) gas mixture (Auciello et al. 2004). The MPCVD process leads to the formation of carbon dimers $\left(\mathrm{C}_{2}\right)$ that provide the main UNCD nucleation species and contribute extensively to the growth process (Carlisle and Auciello 2003). The temperature of the substrate ranged from 400 to $800^{\circ} \mathrm{C}$ and the growth rate was generally $0.1-0.3 \mu \mathrm{m} / \mathrm{h}$ (Carlisle and Auciello 2003).

The silicon and UNCD chips were then treated with a standard piranha solution (1:2 hydrogen peroxide:sulphuric acid in volume) for $20 \mathrm{~min}$ and then rinsed with distilled (DI) water. The UNCD chips then were subjected to a BHF (Buffered Hydrogen Fluoride) oxide etching for about 6 min to remove the PECVD oxide layer and then again rinsed with DI water. They went through a piranha cleaning again to make sure the UNCD chips did not have traces of any chemicals and then again rinsed with DI water. All the chips were finally dried with nitrogen.

\subsection{Contact angle measurements and atomic} force microscopy

The substrate surfaces were characterized by using two different methods, namely: (a) measurement of the water 
Table 1 Surface features of the substrates that are important for cell attachment of various cells

\begin{tabular}{lccll}
\hline & Average height $(\mathrm{nm})$ & RMS roughness $(\mathrm{nm})$ & Contact angles (degrees) & Hydropathy \\
\hline Silicon & 1.0 & $0.4 \pm 0.4$ & $30.4 \pm 3.21$ & Hydrophilic \\
Platinum & 15.8 & $6.1 \pm 0.1$ & $83.6 \pm 2.2$ & Hydrophobic \\
UNCD & 87.8 & $12.6 \pm 1.4$ & $62.4 \pm 2.2$ & Hydrophobic \\
\hline
\end{tabular}

drop contact angle to quantify surface wetability, (b) measurement of surface roughness, via atomic force microscopy (AFM), using a Dimension 5000 scanning probe microscope (SPM, Veeco Instruments, Woodbury, $\mathrm{NY}$ ) in the tapping mode. The images were analyzed using the software WSxM (Nanotec, Spain) (Horcas et al. 2007).

\subsection{Cells and cell culture}

Three phenotypically different cell lines were used to assess biocompatibility of the substrate surfaces studied. These cells were HeLa (cervical carcinoma, epithelial phenotype and obtained from Professor Steve Broyles of Purdue University), PC12 (rat pheochromocytoma cells, neuoprovided by Prof. Riyi Shi of Purdue University), and MC3T3 cells (ATCC, Manassas, VA). The cells were cultured under standard culture conditions of $37^{\circ} \mathrm{C}, 100 \%$ humidity and $5 \% \mathrm{CO}_{2}$ environment in a mammalian culture incubator. The viability and enumeration of all of the cell lines were done by trypan blue staining and it was typically $95 \%$ and never less than $85 \%$ each time. About $1-5 \times 10^{4}$ cells were then seeded on the three substrates separately and incubated for approximately $20 \mathrm{~h}$. The experiments were always run in triplicates.

Dulbecco's modified Eagle medium (DMEM, ATCC) containing 10\% fetal bovine serum (FBS) and antibiotics (penicillin, 1,000 IU/ml and streptomycin, 1,000 $\mu \mathrm{g} / \mathrm{ml}$ ) was used to culture HeLa cells. PC12 cells were cultured in DMEM (GIBCO) with 12.5\% horse serum (Sigma), 2.5\% FBS (Atlanta Biologicals), and antibiotics (penicillin,
Fig. 1 Analysis of the substrate surfaces of Silicon (a), Platinum (b) and UNCD (c). Average heights and RMS surface roughness of the Silicon (blue, left), Platinum (green, middle) and UNCD (red, right) are measured and plotted as topographical histograms (d)
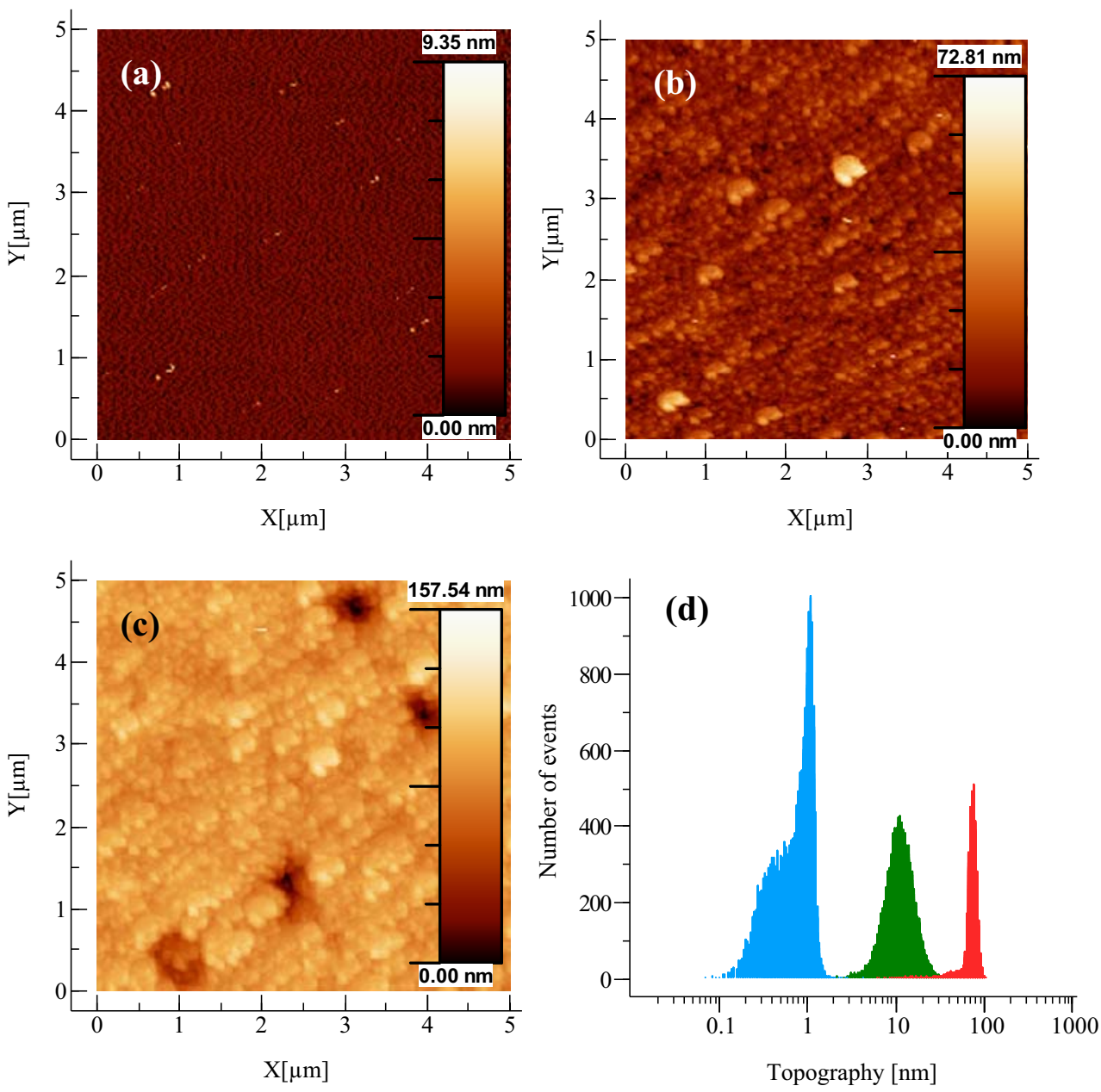
$1,000 \mathrm{IU} / \mathrm{ml}$ and streptomycin, $1,000 \mu \mathrm{g} / \mathrm{ml})$. After culturing the cells in the media described above for 2 weeks, the cells were cultured in the same media supplemented with the NGF $(1 \mu \mathrm{g} / \mathrm{ml})$ in DMEM to convert the cells from a population of replicating chromaffin-like cells to a population of non-replicating sympathetic-neuron-like cells (Greene and Tischler 1982). After overnight culture, the viability of the cells was $96 \%$. Another set of lamininfunctionalized substrates were used for PC12 cells. Silicon, Platinum, and UNCD samples were kept in Hank's balanced salt solution (HBSS)) supplemented with laminin (Sigma, St. Louis, MO) $(10 \mu \mathrm{g} / \mathrm{ml})$ for $18 \mathrm{~h}$ in an incubator. Eagle's medium with alpha modification, supplemented with $10 \%$ FBS and antibiotics (penicillin, 1,000 IU $/ \mathrm{ml}$ and streptomycin, $1,000 \mu \mathrm{g} / \mathrm{ml}$ ) was used to culture MC3T3 osteoblasts. Following the culture of the cells on different substrates, the cells were fixed with glutaraldehyde and rinsed with cacodylate buffer. Then the cell membranes were stained with a lipophilic dye (Octadecylcarboxy cyanine 6-3, Molecular Probes, Eugene, OR) and the cellular nuclei was stained with Hoechst stain and the cells were imaged under a fluorescent microscope with appropriate filters. Finally, the cells were observed by scanning electron microscopy (SEM) to confirm the fluorescent studies.

\subsection{Image analysis}

Fuorescent images of the cells grown on the different substrates were analyzed using ImageJ software (Abramoff et al. 2004). The criteria for the analysis were the numbers of total cells attached, average attached cell areas, average cell rounding and average nuclear area coverage of each cell. The images were adjusted for brightness and contrast and then analyzed with different threshold values to show boundaries of either the cell membranes or nuclei prior to analysis. The number of cells was obtained from the counts of distinct nuclei.

\section{Results and discussions}

\subsection{Surface properties of the substrates}

Surface wetting behaviors of the substrates were analyzed by contact angle measurements. The average contact angles along with the standard deviations are shown in Table 1. The platinum surfaces exhibited an average contact angle of $83.6^{\circ}$ (hydrophobic) and low surface energy, while the silicon surface had a contact angle of $30.4^{\circ}$, (hydrophilic) and high surface energy. The UNCD surface had a contact angle of $62.4^{\circ}$ (light hydrophobic).
Average height and root-mean squared (RMS) surface roughness values as obtained from the AFM analyses for the three different surfaces are also given in Table 1. The AFM image analysis (Fig. 1) indicated that the surface roughness was $0.4 \pm 0.4 \mathrm{~nm}$ for the Si substrate (Fig. 1(a)), $6.1 \pm 0.1 \mathrm{~nm}$ for the platinum/titanium film on Si (Fig. 1(b)), and $12.6 \pm 1.4 \mathrm{~nm}$ for the UNCD film on Si (Fig. 1(c)). The average height histogram distributions of the surfaces indicated that the UNCD surface was the roughest of all of the tested surfaces (Fig. 1(d)).

3.2 Comparison of the interactions between the substrates and the cell types

Interactions of cells from different tissue origin (epithelial, neuronal and osteoblastic) with individual substrate surfaces (Silicon, Platinum, UNCD) were analyzed using both fluorescent images (Fig. 2(a)) and SEMs (Fig. 2(b)). The following criteria were used for the evaluation of in vitro biocompatibility of the surfaces (Fig. 3): total number of the adherent cells, cellular total area coverage, cell rounding and cellular nuclear area coverage. Since cell adhesion is the prerequisite for any further cellular functions such as spreading, migration, proliferation and biosynthetic activity (Chiellini 2006), an increase in the number of cells adhering to a surface indicates that the cells have a greater chance of developing on that surface. The spreading index, which is a function of average cell area per unit cell number, indicates how well the cells will integrate with the host surface (Kirkpatrick et al. 1998). The cell has a circular shape when it is seeded on any surface and the degree to which this shape is changed indicates the affinity to the surface. If a cell is less circular, then there is more interaction between the surface receptors of the cell and the substrates which indicates more spreading and proliferation. Rounding of the cells is one of the indicators of cellular death; hence, we measured cell rounding to quantify the biocompatibility of the surfaces. Another criterion for cell death is condensation or shrinkage of the cellular nuclei hence the average area per nuclei estimates for each substrate surface was obtained from the cells studied.

\subsection{HeLa cell growth on the surfaces}

Results showed a remarkable difference between the numbers of HeLa cells on the three different substrates. The maximum number of cells was observed on the platinum surface, followed closely by the UNCD, and then the silicon surface (Fig. 3(a)). There was a very small difference $(6.45 \%)$ between the number of cells on the $\mathrm{UNCD}$ and the platinum surface indicating that the HeLa cells had a similar affinity for both of these surfaces. 

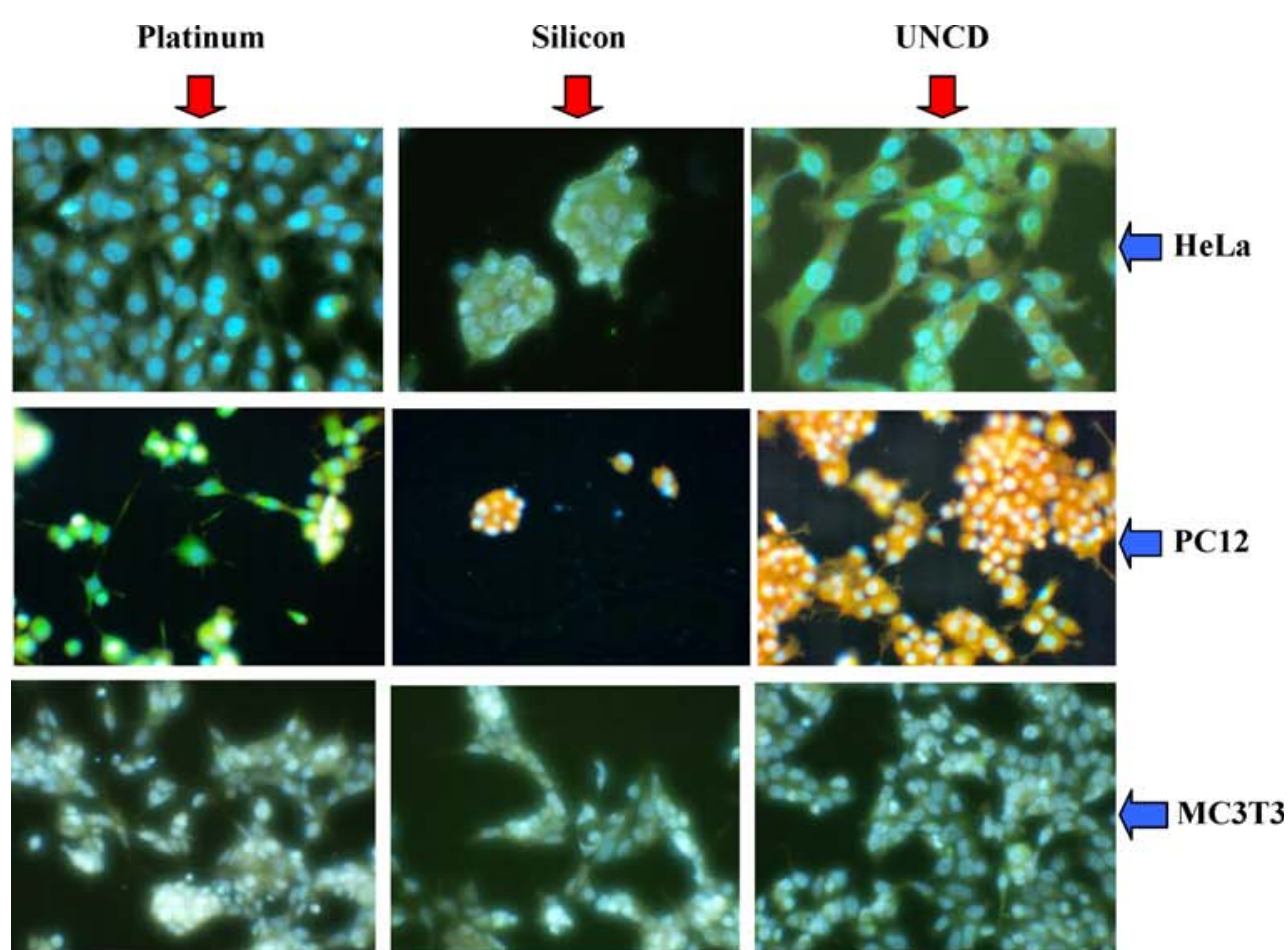

(a)
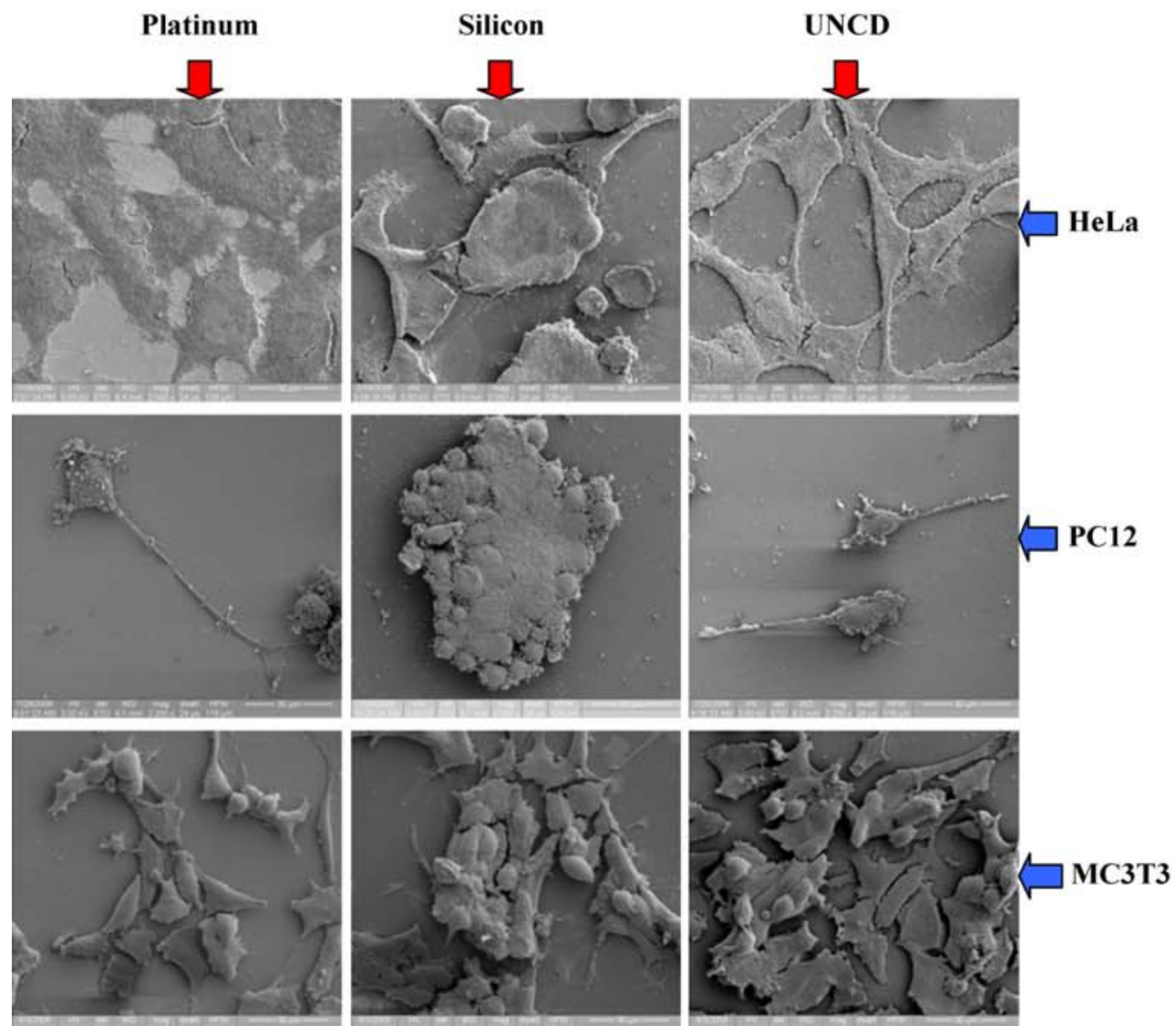

(b)

Fig. 2 Fluorescent microscopic (a) and scanning electron microscopic (b) images of the cell attachment to Platinum ( first column), Silicon (second column) and UNCD (last column). An epithelial cell line, HeLa (top row), neuronal cell line, PC12 (middle row) and osteoblastic

cells (bottom row) were grown $(24 \mathrm{~h})$ on all three surfaces, stained and then imaged. All fluorescence images were acquired at $200 \times$ and SEMs were obtained at $2,000 \times$ magnification 

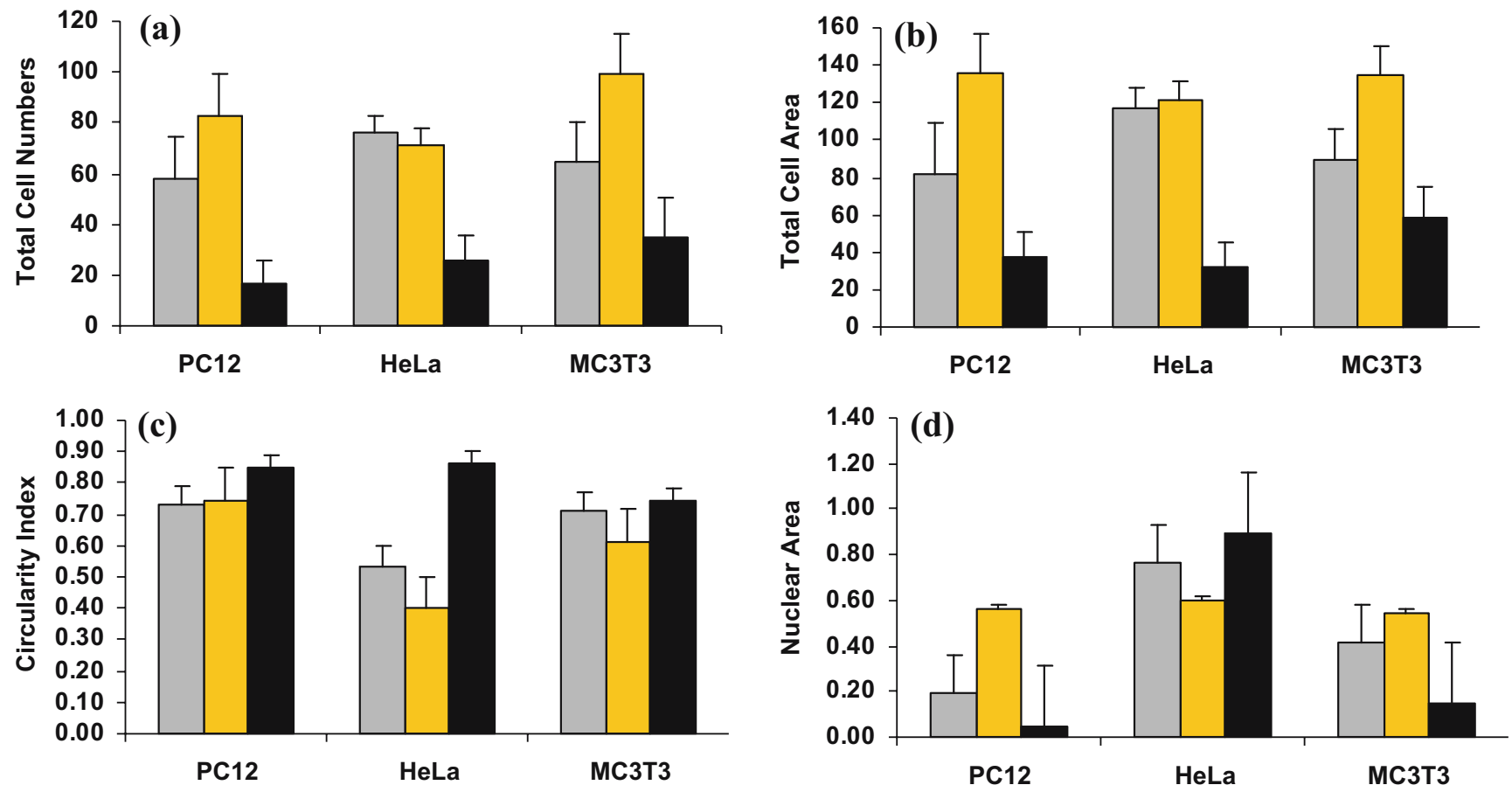

Fig. 3 Quantification of cell attachment and biocompatibility of the Platinum (grey bars, left), UNCD (gold bars, middle) and Silicon (black bars, right) surfaces. Neuronal (PC12), epithelial (HeLa) and osteoblastic (MC3T3) cells were grown on the three unmodified

substrate surfaces and total number of the cells attached to the surfaces (a), total area coverage by each cell and each substrate were determined (b) and cell rounding were quantified (c). The areas of the cellular nuclei in each cell were determined (d)

However, cell spreading is larger on the UNCD surface than on the platinum or silicon surfaces (Fig. 3(b)). The circularity index for the HeLa cells was the best (lowest) for UNCD followed by platinum and silicon (Fig. 3(c)). The silicon surface has fewer cells that clumped together forming islands, indicative of poor cell affinity for the $\mathrm{Si}$ surface. Although the maximum cytoplasmic area from HeLa cells was observed on the UNCD surface, indicating that the cells have a great affinity for this specific material, the cellular nuclei coverage was smaller then the other two surfaces (Fig. 3(d)). Cell spreading was distinctively the lowest on the silicon surface. The results described above indicate that UNCD may provide the best surface for HeLa cells' adhesion, proliferation, and spreading. Silicon on the other hand may not provide an appropriate platform for cell growth, at least for HeLa cells. Platinum shows promise as a better material than silicon, but not equivalent to UNCD.

\subsection{PC12 cell growth on surfaces}

Fluorescence images showed that PC12 cells were growing characteristic neuronal processes and these processes were interacting with each other on both platinum and UNCD surfaces, yet, on the silicon surface they were forming closely packed islands without processes (Fig. 2(a),(b)). This effect can be interpreted as indicative of the affinity of the PC12 cells on Platinum and UNCD surfaces under

study, exhibiting a distinctive outgrowth of axons and dendrites on the surfaces. The maximum PC12 cell numbers (Fig. 3(a)) and spreading (Fig. 3(b)) were again observed on the UNCD surface, followed by the platinum and the silicon surfaces. However, PC12 cells exhibited lower spread (Fig. 2(a),(b)) than the HeLa cells on all three substrates. Vast colonies of cells could be observed in fluorescent images and SEMs clumping up together and exhibiting a circular morphology on Silicon surfaces. It is difficult to isolate $\mathrm{PC} 12$ cells, being neuronal in nature, from the glial cells when seeded for the experiments. The glial cells are supporting cells to the nerve cells and are involved in many functions such as maintaining ionic balances, uptake of neurotransmitters, and recovery from neural injury, but they do not directly participate in synaptic interactions and electrical signal propagation as nerve cells do. Glial cells generally tend to have a circular morphology and they are normally present in close vicinity to each other and to the PC12 cells. The glial cells were also taken into account during the quantitative analysis on the circularity factor. Again, the studies on PC12 cell growth on all three substrates showed that the UNCD surface is the most suitable for PC12 cell attachment (Fig. 3(a)) and growth (Fig. 3(b)). Circularty index of Platinum and UNCD surfaces with PC12 cells were almost equivalent (Fig. 3(c)) yet nuclear area coverage which is another indicator of biocompatibility was the highest on UNCD 
Fig. 4 Fluorescent microscopic analysis of PC12 cell attachment on laminin_coated substrates of Platinum ( (second column) and UNCD (last column). All fluorescence images were acquired at $200 \times$ magnification
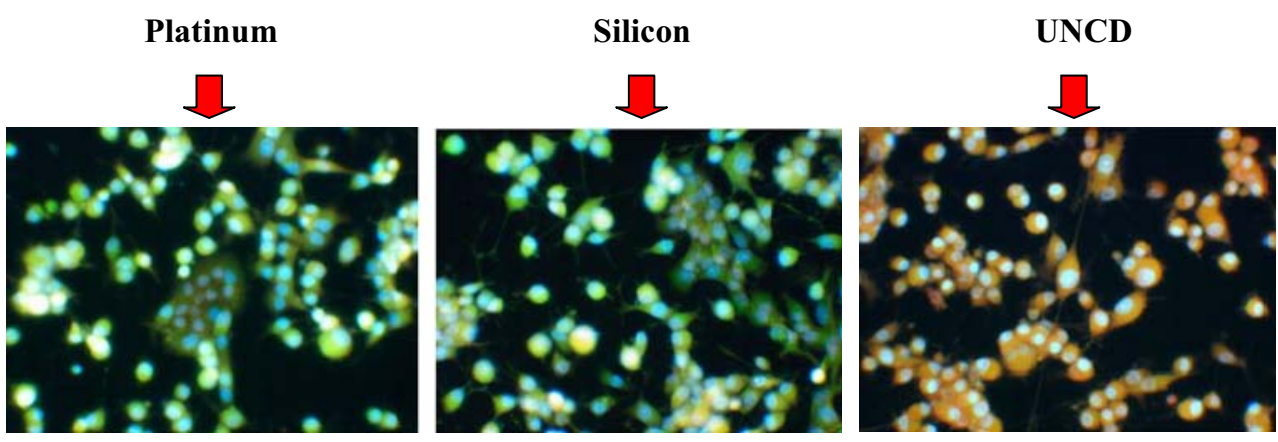

(Fig. 3(d)). Again the Silicon surface was found to be the least biocompatible since circularity index was the highest of the other two surfaces (Fig. 3(c)) and also there was significant shrinkage of the cellular nuclei on Silicon substrates (Fig. 3(d)).

Since neuronal cells generally require cell matrix attachment proteins such as laminin or collagen to attach to inorganic surfaces, studies of neuronal cell growth on all three substrates discussed here were performed, including laminin coating on the substrate surface (Fig. 4). The main reason for the functionalization of the substrate surface was that in practice, the culture of $\mathrm{PC} 12$ cells can only be accomplished via functionalization of the tissue culture plates as these cells are notoriously difficult to culture on the bare substrates. The fluorescence images of functionalized surfaces showed that the maximum number of cells on the surfaces, area, fraction covered with cells, circularity factor, and cell spreading were similar for all three surfaces, while laminin-coated Si substrates showed a slightly better cell interaction for most of these characteristic. These comparisons indicate that after surface functionalization, the substrate does not make a difference for the adhesion of cells. Also, this particular experiment served as a positive control as optimal PC12 cell attachment and growth for the rest of the experiments that were conducted in this study. Attachment studies using the other two cells types on laminin coated surfaces also showed the same results (not shown here).

\subsection{MC3T3 cell growth on surfaces}

Fluorescent microscopic and SEM images showed that maximum number of adherent osteoblastic cells were occurring on the UNCD surface, followed by the platinum and the silicon surfaces, respectively (Fig. 2(a),(b)). As it was occurring with both the HeLa and PC12 cells, osteoblastic phenotype MC3T3 cells were adhering (Fig. 3(a)) and spreading the most on UNCD surfaces (Fig. 3(b)). Quantitative study showed that the cell circularity was lowest on UNCD than the other two surfaces and both Platinum and Silicon had a very similar circularity index (Fig. 3(c)). The nuclear area coverage on the UNCD surface was the highest and both Platinum and Silicon had similar nuclear area coverage with MC3T3 cells (Fig. 3(d)). Since cell adhesion is one of the key properties in determining the affinity of a cell to a substrate, these results indicate that the MC3T3 cells prefer the UNCD surface over platinum and silicon.

\section{Conclusions}

It can be concluded from these studies that UNCD is a promising biocompatible surface as compared to both Silicon and Platinum at a noticeable degree with different types of cells. Maximum cell attachment, cell spreading and nuclear area coverage with all of the tested phenotypically different cells occurred on the UNCD surfaces, indicating that UNCD is the most biocompatible among the platinum and silicon substrates used in this study. Cell rounding was the lowest with UNCD cell substrates as compared with platinum and silicon surfaces with all of the cell lines tested, indicating that UNCD surfaces were not perceived by the cells as cytotoxic. The higher surface roughness of UNCD may be a significant contributing factor for the maximum attachment and growth of cells. Further studies with more cell types are needed to characterize in detail the interaction of UNCD with biological systems.

The high affinity of all cells studied here for UNCD surfaces can be rationalized by considering that the surface of as-grown UNCD films is generally terminated in hydrogen atoms that can provide active chemical bonds for molecules on the surface of the cells. However, more work is needed to confirm this hypothesis and understand the chemistry between the cells and the UNCD surface. In any case, since UNCD films combine some of the most useful properties of single crystalline diamonds like chemical inertness, hardness and a high Young's modulus, but most importantly a surface chemical affinity for biomaterial, it provides a very suitable material platform for manufacturing of sensors and stimulators based on bioMEMS and artificial bio-electronic devices. Inertness of UNCD also implies that it can be used as implantable material since it is not expected to induce a large scale 
immune response in the body. We suggest UNCD as an alternative and potentially very promising material for both fabrication and encapsulation of in vivo devices where biocompatibility and inertness are desired at maximal level.

Acknowledgments We would like to thank Prof. Broyles and Prof. Shi for the providing the different cell lines, and Ms. Laleh Rabieirad of Purdue University for help with the platinum/titanium sputtering. We would like to thank Dr. Hongjun Zeng of Nanotechnology Core Facility of University of Illinois, Chicago for performing the PECVD of the silicon dioxide on the UNCD substrate. We would like to thank INAC program at Purdue for providing the funding to support this study. ANL researchers acknowledge support from the US Department of Energy, BES-Materials Sciences, under Contract DE-AC02-06CH11357.

\section{References}

M.D. Abramoff, P.J. Magelhaes, S.J. Ram, Biophoton. Int. 11, 36 (2004)

O. Auciello, A.R. Krauss, D.M. Gruen, H.G. Busmann, E.M. Meyer, J. Tucek, A. Sumant, A. Jayatissa, N. Moldovan, D.C. Mancini, M.N. Gardos, MRS Symposium Proceedings 605, 73 (2000)

O. Auciello, J. Birrell, J.A. Carlisle, J.E. Gerbi, X. Xiao, B. Peng, H.D. Espinosa, J. Phys. Condens. Matter 16, 539 (2004)
J.A. Carlisle, O. Auciello, Interface 12, 28 (2003)

F. Chiellini, J. Bioact. Compat. Polym. 21, 257 (2006)

R.A. Freitas Jr., Nanomedicine, Volume IIA: Biocompatibility, (Landes Bioscience, Georgetown, 2003), p 183, 185

M.N. Gardos, Surf. Coat. Technol. 113, 183 (1999)

L.A. Greene, A.S. Tischler, Adv. Cell. Neurobiol. 3, 373 (1982)

D.M. Gruen, S. Liu, A.R. Krauss, X. Pan, J. Appl. Phys. 75, 1758 (1994a)

D.M. Gruen, S. Liu, Krauss, A.R. Krauss, J. Luo, X. Pan, Appl. Phys. Lett. 64, 1502 (1994b)

D.M. Gruen, D.G. Zuiker, A.R. Krauss, X. Pan, J. Vac. Sci. Technol. A 13, 1628 (1995)

C. de Haro, R. Mas, G. Abadal, J. Muñoz, F. Perez-Murano, C. Domiñguez, Biomaterials 23, 4515 (2002)

I. Horcas, R. Fernández, J.M. Gómez-Rodríguez, J. Colchero, J. Gómez-Herrero, A.M. Baro, Rev. Sci. Instrum. 78, 0137051 (2007)

S. Jiao, A. Sumant, M.A. Kirk, D.M. Gruen, A.R. Krauss, O. Auciello, J. Appl. Phys. 90, 118 (2001)

C.J. Kirkpatrick, F. Bittinger, M. Wagner, H. Köhler, T.G. van Kooten, C.L. Klein, M. Otto, J. Eng. Med. 212, 75 (1998)

H.J. Steffen, J. Schmidt, A.G. Elipe, Surf. Int. Anal. 29, 386 (2000)

X. Xiao, J. Wang, J.A. Carlisle, B. Mech, R. Greenberg, R. Freda, M. S. Humayun, J. Weiland, O. Auciello, J. Biomed. Mater. 77B2, 273 (2006)

W. Yang, O. Auciello, J.E. Butler, W. Cai, J.A. Carlisle, J.E. Gerbi, D. M. Gruen, T. Knickerbocker, T. Lasseter, J.N. Russell, L.M. Smith, R.J. Hamers, Nat. Mater. 1, 253 (2002) 